Pacific Journal of Mathematic 


\title{
GENERATING IDEALS IN PRÜFER DOMAINS
}

\author{
RAYMOND C. HeITMANN
}

There is a recent theorem that finitely generated ideals in a Prüfer domain of Krull dimension one can be generated by two elements. Whether or not this property holds in Prüfer domains without the dimension assumption is still undecided. However, in this paper, a dimension-dependent bound on the number of generators is derived. Precisely, a finitely generated ideal in an $n$-dimensional Prüfer domain can be generated by $n+1$ elements. In fact, the bound actually holds for invertible ideals in any domain.

A number of related results also appear. Several theorems previously known only for commutative rings with noetherian maximal spectrum have been extended to Prüfer domains. These include Serre's Theorem (concerning free summands of projective modules) and a (projective modules only) version of the Forster-Swan Theorem. Also, Bass's Stable Range Theorem is extended to the case of arbitrary domains. If zero-divisors are allowed, bounds increase by one.

The 1-dimensional result of Vasconcelos and Sally [7] was the first positive result on the "2-generator question". This suggested to L. Levy the possibility of attaining a dimension dependent bound. While the proof could not be extended, the natural bound has been shown to hold. Upon seeing the initial proof of the main result, R. Wiegand suggested it might be applicable to some of the noetherian spectrum theorems. Although the goal of a uniform approach to these theorems was not attained, the efforts in this direction did meet with some success. Also, the dimension of the entire prime spectrum is used throughout rather than the (lower) dimension of the maximal spectrum. This seems to be a casualty of dropping the noetherian assumption - the possibility of a sharper bound is discussed in $\S 4$.

Section 1 is devoted to the demonstration of a few elementary lemmas concerning generating elements for locally principal ideals. Section 2 introduces the machinery needed to prove the main theorem and the other results. Therein, $R$ must be a Prüfer domain primarily to force primes contained in a given prime to be linearly ordered. A notationally complex, and consequently somewhat obscure, method of removing this hypothesis is devised in $\$ 2^{*}$. This section may be omitted without loss of continuity on a first reading. Section 3 states and proves all of the major theorems, and $\$ 4$ is devoted to statements and questions with regard to possible improvements. 
Notation. $R$ will always denote a commutative ring. The symbols $P$ and $Q$ will be reserved for primes of $R$. The symbols $I$ and $J$ and the term ideal will always designate finitely generated nonzero ideals.

It is useful to recall some basic properties of Prüfer domains. Localizations at primes are valuation rings. (Finitely generated) ideals are projective, invertible, and locally principal. Also, the element $x$ generates $I$ at $P$ if and only if $x I^{-1} R_{P}=R_{P}$.

1. Closed sets and generating elements. In this section, it is assumed that the ideal $I$ is locally principal. As this paper only endeavors to find generating sets for invertible ideals, this shall cause no difficulty. The goal here is to find one common generator for $I$ on an entire closed set of primes. Spec $R$, the usual topological space will be employed. Recall that the points in this space are the primes and closed sets $V(A)$ are all those primes which contain $A$, a subset of $R$. (Each subset determines a closed set.)

Lemma 1.1. If $B_{1}, B_{2}$ are disjoint closed sets in $\operatorname{Spec} R$, then there exists

$$
a \in \bigcap_{P \in B_{1}} P-\bigcup_{P \in B_{2}} P
$$

Proof. If $B_{1}=V\left(A_{1}\right) ; B_{2}=V\left(A_{2}\right) ;$ then $A_{1} R+A_{2} R=R$. This enables us to write $1=a_{1}+a_{2}$ for some $a_{1} \in A_{1} R$ and $a_{2} \in A_{2} R$. It is easy to see that $a_{1}$ is the desired element.

Lemma 1.2. If $B_{1}, B_{2}$ are disjoint closed sets in $\operatorname{Spec} R$; and a generates $I$ on $B_{1}$, then there exists $a^{\prime} \in I$ which generates $I$ on $B_{1}$, but generates at no prime of $B_{2}$.

Proof. By a generates $I$ on $B_{1}$, we mean locally generates at each prime of $B_{1}$.

This property won't be disturbed if we multiply by an $r \in R$ which is locally a unit at these primes. So, by (1.1), pick $r \in \bigcap_{P \in B_{2}} P-\bigcup_{P \in B_{1}} P$, and $a^{\prime}=r a$ works.

LEMMA 1.3. If $A, B_{1}, \cdots, B_{n}$ is a finite family of disjoint closed sets in Spec $R$; and $a_{1}$ generates $I$ on $B_{1}$, then there exists $a^{\prime} \in I$ which generates on $\cup B_{1}$ but at no prime of $A$.

Proof. By (1.2), we can assume that $a_{i}$ generates at no primes in $A \cup \cup_{i \neq j} B_{i}$. Then $a^{\prime}=\sum a_{i}$ is the desired element (because generator + nongenerator $=$ generator, locally). 
These lemmas are quite trivial, but are quite useful provided we can find disjoint closed sets, on each of which the ideal has a generator. In the next section, several such will be described. Others, yet undiscovered, might lead to new results.

\section{Useful closed sets.}

Definition 1. Let $R$ be a Prüfer domain; $P$ a fixed prime; then define

$$
\begin{aligned}
& I \equiv_{P} J \Leftrightarrow(\operatorname{rad} I) R_{P}=(\operatorname{rad} J) R_{P} \\
& I \ll_{P} J \Leftrightarrow(\operatorname{rad} I) R_{P} \varsubsetneqq(\operatorname{rad} J) R_{P}
\end{aligned}
$$

$(\operatorname{rad} I)$ of course denotes the prime radical of $I$.

\section{LEMMA 2.1. For any pair $(I, J),\left\{P \mid I \ll_{P} J\right\}$ is closed.}

Proof. This set is just the support of the finitely generated module $J /(\operatorname{rad} I \cap J)$ and so must be closed [1, p. 107, Prop. 17].

Definition 2. Let $\mathscr{A}$ denote a finite collection of ideals; define $m(P, \mathscr{A})$ to be the largest attainable length of a strict inequality $\left(\ll_{P}\right)$ chain of ideals in $\mathscr{A}$.

From the definition of $\ll_{P}$, it is immediately seen that $m(P, \mathscr{A}) \leqq$ $\operatorname{dim} R_{P}$ for every $\mathscr{A}$. Also, if $R$ has Krull dimension $n$, this uniformly yields $m(P, \mathscr{A}) \leqq n$ for every pair $(P, \mathscr{A})$.

Definition 3. For primes $P$ and $Q$ and a collection of ideals $\mathscr{A}$, define $P \sim_{\mathscr{A}} Q \Leftrightarrow \ll_{P}$ and $\ll_{Q}$ agree on $\mathscr{A}$.

Proposition 2.2. Let $R$ be a Prüfer domain; $\mathscr{A}$ a finite collection of ideals. Then

(i) $m(-, \mathscr{A})$ is upper semi-continuous on Spec $R$.

(ii) If $A$ is closed in Spec $R$ and $m(P, \mathscr{A}) \leqq k$ for all $P \in A$, then $C=\{P \in A \mid m(P, \mathscr{A})=k\}$ is closed and each $\left(\sim_{\mathscr{A}}\right)$ equivalence class of $C$ is closed.

Proof. (i) Suppose $B=\{Q \in \operatorname{Spec} R \mid m(Q, \mathscr{A}) \geqq k\}$. Let $B_{1}, \cdots, B_{n}$ be the $\left(\sim_{A}\right)$ equivalence classes of $B$. By $(2.1), \bar{B}_{i} \subset B$ for all $i$; so $\bar{B}=B$. (Note that $\mathscr{A}$ finite implies $\operatorname{Spec} R / \sim_{\mathscr{A}}$ finite.)

(ii) Let $C_{1}, \cdots, C_{n}$ be the equivalence classes of $C$. It will suffice to prove that $C_{1}$ is closed. If $P \in C_{1}$, there are ideals $I_{0}, \cdots, I_{k}$ in $\mathscr{A}$ such that $I_{0} \ll_{P} \cdots I_{k}$ and every ideal in $\mathscr{A}$ is $\equiv_{P}$ to some $I_{i}$. Now, if $Q \in \overline{C_{1}}$, 
$Q \in A$ as $A$ is closed. Further, (2.1) yields $I_{0} \ll_{O} \cdots I_{k}$. If $Q \notin C_{1}$, then there must exist $J \in \mathscr{A}$ where $J \equiv_{P} I_{l}$ but $J \ll_{O} I_{l}$ (or $I_{i} \ll_{Q} J$, yielding identical proof). By (2.1), $I_{i-1} \ll_{Q} J$ and so $I_{0} \ll_{Q} \cdots I_{i-1} \ll_{Q} J \ll_{Q}$ $I_{i} \ll_{Q} \cdots I_{k}$. Thus $m(Q, \mathscr{A})>k$, a contradiction.

Lemma 2.3. (Let $R$ be a Prüfer domain.) If $\left\{x_{i}\right\}$ is a finite generating set for an invertible ideal $I$, and $\mathscr{A}$ contains $x_{1} I^{-1}$ for each $i$, then I may be generated on $C$ (defined as in (2.2.)) by one element.

Proof. Because $\left\{x_{i}\right\}$ generates $I$, for each $P$ there is an $x_{i}$ such that $x_{t} I^{-1} R_{P}=R_{P}$ ( $I$ being invertible). Further, this equation holds for $P$ if and only if it holds for every prime in the $\left(\sim_{\mathscr{A}}\right)$ equivalence class of $P$. Hence, all of the primes in a given class have a common generator. So, $C$ is a finite union of disjoint closed sets, each of which has a generator. (1.3) completes the proof.

Note. The hypothesis that $R$ must be a Prüfer domain in (2.3) is a carry-over from the hypothesis of (2.2); it is otherwise not required. Consequently, when the hypothesis is effectively removed from the earlier proposition in the next section, it is seen to be superfluous here. For this reason, it has been denoted parenthetically.

2*. More generality. The crux of $\$ 2$ was the value in inducing closed sets of the inequality $\ll_{p}$. Strict inequality chains corresponded to chains of primes (except $R$ was included and zero excluded). So finite Krull dimension bounded the length. When the Prüfer hypothesis is dropped, the situation becomes more difficult. However, while the transitivity of the inequality must be sacrificed, the basic idea still works. So new definitions are given, properly generalizing those of $\S 2$.

DefinItION 1. Let $I_{0}, \cdots, I_{n}$ be ideals of $R$. Inductively define subsets $E(\quad)$ of $\operatorname{Spec} R$ by

(i) $E\left(I_{0}\right)=\operatorname{Spec} R$

(ii) $E\left(I_{0}, \cdots, I_{n}\right)=$ closure $\left\{E\left(I_{0}, \cdots, I_{n-1}\right) \cap V\left(I_{n-1}\right) \cap D\left(I_{n}\right)\right\}$.

Observation. When $R$ is Prüfer, $P \in E\left(I_{0}, \cdots, I_{n}\right) \Leftrightarrow I_{0} \ll_{P} \cdots I_{n}$.

Observe that the set in brackets is the intersection of a closed set and a quasi-compact open set. Hence it is a patch (A patch is a subset of Spec $R$ closed in the patch topology - whose closed sets are generated by the closed and the quasi-compact open sets, i.e., $D(I)$ for finitely generated $I$, of $\operatorname{Spec} R)$ and $E\left(I_{0}, \cdots, I_{n}\right)$ is the closure of a patch. As the closure of a patch is the union of its pointwise closures [5, p. 45], we 
see that $P \in E\left(I_{0}, \cdots, I_{n}\right) \Leftrightarrow$ there exists $Q \subseteq P$ such that $Q \in E\left(I_{0}, \cdots, I_{n-1}\right), I_{n-1} \subseteq Q$, and $I_{n} \not \subset Q$.

NoTE. Careful inspection of this condition yields a correspondence, as before, between $P \in E\left(I_{0}, \cdots, I_{k}\right)$ and a prime chain of length $(k-1)$ contained in $P$. This suggests:

Definition 2. For a finite collection of ideals $\mathscr{A}$, set $m(P, \mathscr{A})$ equal to the highest integer $k$ such that there exist $I_{0}, \cdots, I_{k} \in \mathscr{A}$ giving $P \in E\left(I_{0}, \cdots, I_{k}\right)$.

By the above note, it is seen that if $R$ is any domain, $m(P, \mathscr{A}) \leqq$ dimension $R$. If the domain hypothesis is deleted, minimal primes can occur in the chains (and $R$ as well). Here, then, $m(P, \mathscr{A}) \leqq \operatorname{dim} R+1$ holds. Throughout, this extra 1 will appear in the nondomain case.

Definition 3. For primes $P, Q$ and a collection of ideals $\mathscr{A}$ $P \sim{ }_{\mathscr{A}} Q \Leftrightarrow$ for each ordered subset of $\mathscr{A}, P \in E\left(I_{0}, \cdots, I_{k}\right)$ if and only if $Q \in E\left(I_{0}, \cdots, I_{k}\right)$.

Proposition 2.2*. Let $R$ be a commutative ring; $A$ a finite col ection of ideals which generate $R$. Then

(i) $m(-, \mathscr{A})$ is upper semi-continuous on Spec $R$.

(ii) If $A$ is closed in Spec $R$ and $m(P, \mathscr{A}) \leqq k$ for all $P \in A$, then $C=\{P \in A \mid m(P, \mathscr{A})=k\}$ is closed and expressible as a finite disjoint union of closed sets $\left\{C_{i}\right\}$ such that for each $i$, there is an $I \in \mathscr{A}$ satisfying $I R_{P}=R_{P}$ for all $P \in C_{i}$.

Proof. (i) Suppose $B=\{Q \in \operatorname{Spec} R \mid m(Q, \mathscr{A}) \geqq k\}$. Let $\dot{B}_{1}, \cdots, B_{n}$ be the $\left(\sim_{A}\right)$ equivalence classes of $B$. As the sets $E(\quad)$ are closed, $\bar{B}_{i} \subset B$ for all $i$. So $\bar{B}=B$.

(ii) For each prime $P$, the set of ideals in $\mathscr{A}$ satisfying $I R_{P}=R_{P}$ form a subset (nonempty by hypothesis). If two primes induce the same subset, call them congruent. The finiteness of $\mathscr{A}$ guarantees that the number of congruence classes is finite. Let $\left\{C_{i}\right\}$ be the set of congruence classes of $C$. Then the proof will be complete provided each $C_{i}$ is closed.

It suffices to consider $C_{1}$. By (i) and the fact that $A$ is closed, $C$ is closed, yielding $\overline{C_{1}} \subset C$. Designate those ideals of $\mathscr{A}$ not contained in $P$, for $P \in C_{1}$, by $J$ and those contained in $P$ by $I$. The result follows if $Q \in \bar{C}_{1}$ implies $J \not \subset Q$ and $I \subset Q$.

Now observe $C_{1} \subset E(I, J)$; hence $Q \in E(I, J)$ and $I \subset Q$. Let $D$ be one of the finitely many $\sim_{\mathscr{A}}$ equivalence classes of $C_{1}$. The assumption $m(P, \mathscr{A})=k$ implies the existence of ideals $I_{0}, \cdots, I_{k-1}$ in $\mathscr{A}$ such that 
$D \subset E\left(I_{0}, \cdots, I_{k-1}, J\right)$. If $D$ was chosen so that $Q \in E \bar{D}$, then $Q \in$ $E\left(I_{0}, \cdots, I_{k-1}, J\right)$. Now it is claimed that $J \not \subset Q$. For otherwise, some $J_{1} \not \subset Q$ and it would follow that $Q \in E\left(I_{0}, \cdots, I_{k-1}, J, J_{1}\right)$ - a contradiction.

\section{Results.}

THEOREM 3.1. Let $R$ be an $n$-dimensional commutative domain containing an invertible ideal $I$. Then $I$ may be generated by $n+1$ elements, the first of which may be chosen arbitrarily. If $R$ is not a domain, I requires $\leqq n+2$ generators.

Proof. Pick $\mathscr{A}$ in accordance with the hypothesis of (2.3). Generators $y_{n}, \cdots, y_{1}$ will be inductively defined as follows:

Set $A_{k}=$ support $\left(I /\left(y_{k+1}, \cdots, y_{n}\right)\right)$, the closed set of primes on which the set $\left\{y_{k+1}, \cdots, y_{n}\right\}$ does not generate $I$. $\left(A_{n}=\operatorname{Spec} R\right)$ Assume $m(P, \mathscr{A}) \leqq k$ on $A_{k}$. (This is true for $k=n$ by the dimension hypothesis.) Let $C_{k}=\left\{P \in A_{k} \mid m(P, \mathscr{A})=k\right\}$ as in (2.2). Next employ (2.3) to select $y_{k}$, which generates $I$ on $C_{k}$. Hence $C_{k} \cap A_{k-1}=\varnothing$ and $m(P, \mathscr{A}) \leqq k-1$ on $A_{k-1}$ as needed.

Finally, on $A_{0}, m(P, \mathscr{A})=0$ and every member of the initial generating set locally generates $I$. So let $y_{0}$ be an arbitrary initial generator. Then $I=\left(y_{n}, \cdots, y_{0}\right)$ as desired.

If $R$ is not a domain, the dimension hypothesis only asserts $m(P, \mathscr{A}) \leqq n+1$ and so an additional generator is required.

Similarly we obtain a projective analogue of the Forster-Swan Theorem (3.2) and, as a corollary to that proof, Serre's Theorem is extended to Prüfer domains (3.3).

THEOREM 3.2. If $R$ is an $n$-dimensional Prüfer domain and $M$ is a rank d projective $R$-module, then $M$ may be generated by $n+d$ elements.

Proof. $\quad M$ is isomorphic to a direct sum of $d$ ideals [2, p. 14, Prop. 6.1] because $R$ is Prüfer. (This is actually the only place the Prüfer hypothesis is needed.) So suppose $M=\bigoplus_{i=1}^{d} I_{i}$. Let $\left\{x_{i j}\right\}$ be a finite generating set for $I_{i}$. Then set $\mathscr{A}=$ the set consisting of $x_{i j} I_{i}^{-1}$ for all $i, j$. A satisfies the hypothesis of (2.3) with respect to each of these ideals and so a (slightly modified) version of the proof of (3.1) may be used to find $n+1$ element generating sets for these ideals.

Generating sets for $I_{1}, \cdots, I_{d}$ are derived, one ideal at a time. The generating set for $I_{i}$ will be denoted $y(i, n), \cdots, y(i, 0)$, being inductively defined in that order. The procedure, much as before (identical for $I_{1}$ ), follows:

Set $A(i, k)=$ support $\left(I_{1} /(y(i, k+1), \cdots, y(i, n))\right)$. Assume that 
$m(P, \mathscr{A}) \leqq k$ for each $P \in A(i, k)$ (The dimension assumption gives this for $k=n$ ), and $A(i-1, k) \subset A(i, k)$ (trivial for $k=n$ ). Then set $C(i, k)=\{P \in A(i, k) \mid m(P, \mathscr{A})=k\}$. Now observe that $A(i-1, k-1)$ $\cap C(i, k)=\varnothing$ because the function $m(-, \mathscr{A})$ has conflicting values on the two sets. Consequently, by $(2.3)$ and $(1.3), y(i, k)$ may be selected to locally generate $I_{i}$ on $C(i, k)$ but at no prime of $A(i-1, k-1)$.

Generation on $C(i, k)$ and the induction assumption give $m(P, \mathscr{A}) \leqq$ $k-1$ on $A(i, k-1)$; and nongeneration on $A(i-1, k-1)$ plus the corresponding induction assumption (and the obvious $A(i, k-1) \subset$ $A(i, k))$ yield $A(i-1, k-1) \subset A(i, k-1)$ as needed. At the termination of the induction $(k=1)$, again select $y(i, 0)$ arbitrarily from the initial set of generators.

At the termination of the entire process, each ideal $I_{i}$ (Make no distinction between the ideal and submodule of $M$. Think of $y(i, k)$ as an element of $M$.) has a set of generators $\{y(i, k)\}$. Further, the containment relations forced upon the $A$ 's gives " $i<j$ implies $A(i, k) \subset A(j, k)$ ". To utilize this condition, it is convenient to define $k(i, p)$ to be the least integer $k$ such that $P \in A(i, k)$, i.e., the greatest integer $k$ such that $y(i, k)$ generates $I_{i}$ at $P$. The condition then becomes " $i<j$ implies $k(i, P) \geqq k(j, P)$ ". Now consider the matrix where the $i$ th row is a generating set for $I_{i}$.

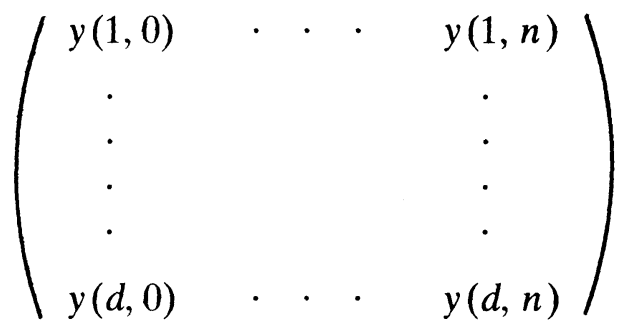

Consider all of the "diagonals" of this matrix with $i-j=$ constant. Each element lies on exactly one of these and there are $d+n$ in all. Summing all of the elements on each "diagonal" gives $n+d$ new elements which, it will be shown, are a generating set for $M$.

So let $M^{\prime}$ be the module these elements generate. It is enough to show $M^{\prime}=M$ locally and by Nakayama's Lemma, it is actually sufficient to show $M \subseteq M^{\prime}+M P$. This can be achieved by the following inductive step:

$$
\text { “ } \bigoplus_{i<1} I_{\imath} \subset M^{\prime}+M P \quad \text { implies } \quad I_{J} \subset M^{\prime}+M P "
$$

Let $y$ be the generating element of $M^{\prime}$ which contains the summand $y(j, k(j, P))$. All summands of $y$ have the form $y(j+h, k(j, P)+h)$. For $h<0$, these elements lie in $\bigoplus_{1<j} I_{i}$ and so also in $M^{\prime}+M P$ by the 
induction assumption. For $h>0$, the condition on, the $k$-function asserts

$$
k(j+h, P) \leqq k(j, P)<k(j, P)+h
$$

and so each of these is in $M P$. Thus $y(j, k(j, P)) \in M^{\prime}+M P$ and this element is a local generator for $I_{j}$. Hence $M^{\prime}=M$ as desired.

Corollary 3.3. If $R$ is an $n$-dimensional Prüfer domain, and $M$ is projective of rank $(n+1)$, then $M$ has a free summand.

Proof. Let $M \cong \oplus I_{i}$ and find generators $\{y(i, j)\}$ exactly as in (3.2). Then we claim $y=\sum_{i+\jmath=n+1} y(i, j)$ generates a free summand of $M$.

Fix a prime $P$. The first observation is that one of the $y(i, j)$ 's locally generates the corresponding $I_{i}$ at $P$. (This is a trivial consequence of the inclusion relation we placed on the $A$ 's.) Hence $y$ and $n$ other elements generate the free $R_{P}$-module $M_{P}$. By [3, p. 283, L. 1], $y$ generates a free direct summand of $M$.

Note. Because $M \oplus L \cong N \oplus L$ implies $M \cong N$ for projective modules over Prüfer domains [6, p. 75], (3.2), which is equivalent to the statement that every projective has a rank $n$ complement (summing to a free), actually implies (3.3) directly.

Because the ring itself is always invertible, the Stable Range Theorem requires no Prüfer assumption. So, in full generality

THEOREM 3.4. (Stable Range Theorem) If $R$ is an $n$-dimensional commutative domain, and $\left(b_{1}, \cdots, b_{k}\right)$ generates $R$ with $k>n+1$, then there exists $\left\{r_{1}\right\}_{1=1}^{k-1}$ such that $\left(b_{1}+r_{1} b_{k}, \cdots, b_{k-1}+r_{k-1} b_{k}\right)$ also generates $R$. Furthermore, if $k>n+2, R$ need not be a domain.

Proof. By an ordinary telescoping argument, it suffices to find a set $\left\{s_{i} \mid 1 \leqq i \leqq k-1\right\}$ such that $\left\{b_{i}+s_{i} b_{i+1}\right\}$ is a generating set. In fact, we then set

$$
r_{\imath}=(-1)^{i+k-1} \cdot \prod_{j=i}^{k-1} S_{j}
$$

and obtain the theorem.

The $\left\{s_{l}\right\}$ will be defined by an inductive procedure beginning with $i=k-1$ and descending. Call $\left(b_{1}, \cdots, b_{k}\right)$ the $k$ th sequence and let $A_{k-1}=\operatorname{Spec} R$. At the $i$ th step, assume the $(i+1)^{\text {st }}$ sequence generates $R$ (true for $i=k-1$ ). 
Set $A_{i}=\operatorname{Support}\left(R /\left(b_{i+1}+s_{i+1} b_{i+2}, \cdots, b_{k-1}+s_{k-1} b_{k}\right)\right)$.

Assume that $A_{i} \cap E\left(\left(b_{1}\right), \cdots,\left(b_{l+1}\right)\right)=\varnothing$. (For $i=k-1$, the dimension hypothesis forces the second set to be empty.)

$$
\text { Set } B_{i}=A_{i} \cap E\left(\left(b_{1}\right), \cdots,\left(b_{i}\right)\right) \text {. }
$$

$B_{i}$ is closed and it may be shown that $b_{i} \notin P$ for $P \in B_{i}$. To see this, observe some member of the $(i+1)^{\text {st }}$ sequence is not in $P$. As $P \in A_{i}$, this implies $b_{1} \notin P$ for some $j \leqq i+1$. $P \in E\left(\left(b_{1}\right), \cdots,\left(b_{1}\right)\right)$ implies $b_{j} \in P$ for $j<i$. Now if $b_{\imath} \in P$ and $b_{\imath+1} \notin P$, then $P \in E\left(\left(b_{1}\right), \cdots,\left(b_{i+1}\right)\right)$, a set disjoint from $A_{i}$. So $b_{i} \notin P$.

$$
\text { Set } C_{\iota}=\operatorname{support}\left(R /\left(b_{1}, \cdots, b_{\imath}, b_{i+1}+s_{i+1} b_{\imath+2}, \cdots, b_{k-1}+s_{k-1} b_{k}\right)\right) .
$$

$B_{i}$ and $C_{1}$ are disjoint closed sets. Hence there is an element $s_{i}$ which is a unit locally at primes of $C_{i}$ and a nonunit at primes of $B_{i}$. Trivially, $b_{\imath}+s_{i} b_{t+1}$ is a unit at primes of $B_{i} \cup C_{l}$. So $B_{\imath} \cap A_{i-1}=\varnothing$ and consequently $A_{t-1} \cap E\left(\left(b_{1}\right), \cdots,\left(b_{i}\right)\right)=\varnothing$. Further, if we define the $i$ th sequence by replacing $b_{i+1}$ with $b_{\imath}+s_{\imath} b_{\imath+1}$, this sequence generates $R$ because the new element is a local generator on primes of $C_{i}$. So we have both induction assumptions.

At the completion of the " $i=1$ " step, the induction gives $A_{0} \cap$ $E\left(\left(b_{1}\right)\right)=\varnothing . \quad$ As the latter $\operatorname{set}=\operatorname{Spec} R, \quad A_{0}=\varnothing . \quad$ So $\left(b_{1}+s_{1} b_{2}, \cdots, b_{k-1}+s_{k-1} b_{k}\right)$ generates $R$ as desired.

As usual, if $R$ is not a domain, the initial assertion that $E\left(\left(b_{1}\right), \cdots,\left(b_{k}\right)\right)=\varnothing$ requires $k>n+2$ - the reason for the higher bound.

4. Extensions, generalizations, comments. As noted in the introduction, it would be preferable if the dimension of the maximal spectrum could be used instead of the Krull dimension in order to bring these theorems into line with the noetherian spectrum theorems. This does not seem entirely possible. However, some improvement can be made. Noting that dimension was only used to bound $m(P, \mathscr{A})$, recall the version of Definition 1 in $\$ 2 *$ :

$$
E\left(I_{0}, \cdots, I_{n}\right)=\text { closure }\left\{E\left(I_{0}, \cdots, I_{n-1}\right) \cap V\left(I_{n-1}\right) \cap D\left(I_{n}\right)\right\} .
$$

To use this, advantage was taken of the fact that the set in brackets was a patch and also the fact that an ideal which generated the ring locally was in fact all of $R$. The same holds true if the entire prime spectrum is replaced by the smallest patch containing the maximal spectrum (the 
closure of the maximal spectrum in the patch topology). Call the dimension of this patch the $p$-dim. Then clearly

$$
\begin{aligned}
& m(P, \mathscr{A}) \leqq p-\operatorname{dim} \text { if }(0) \text { is in the patch. } \\
& m(P, \mathscr{A}) \leqq p-\operatorname{dim}+1 \text { if }(0) \text { is not in the patch. }
\end{aligned}
$$

In the second case, the Jacobson radical is nonzero if $R$ is a domain. There, the arbitary generator implies a further reduction in the bound. Now, as the maximal spectrum is a patch in Spec $R$ when it is noetherian [8], this notion is in fact very closely related to existing results. Thus:

THEOREM 4.1. If the $j$-spectrum of $R$ is a patch in $\operatorname{Spec} R$, then Krull dim. may be replaced by j-dim. in the hypotheses of (3.1), (3.2), (3.3), and (3.4). However, we can no longer select a generator arbitrarily in the case of nonzero radical.

It would be nice to see what happens if $M$ is not a projective module. Can any of the noetherian spec theorems be extended to the Prüfer case? I have not endeavoured to prove these theorems for a finitely generated algebra $A$ over $R$ and have no opinion on the validity of those extensions. We would also like a uniform treatment of (3.2) and (3.4) (as in [3, Theorem B]), but this result has escaped.

The author wishes to acknowledge numerous helpful suggestions offered by the referee which substantially improved the presentation.

\section{REFERENCES}

1. N. Bourbaki, Commutative Algebra, Hermann, Paris, 1972.

2. H. Cartan and S. Eilenberg, Homological Algebra, Princeton University Press, Princeton, 1956.

3. D. Eisenbud and E. G. Evans, Generating modules efficiently: theorems from algebraic $K$-theory, J. Algebra, 27 (1973), 278-305.

4. R. Heitmann and L. Levy, $1-1 / 2$ and 2 Generator Ideals in Prüfer Domains, Rocky Mountain J., 5 (1975), 361-373.

5. M. Hochster, Prime ideal structure in commutative rings, Trans. Amer. Math. Soc., 142 (1969), 43-60.

6. I. Kaplansky, Infinite Abelian Groups, rev. ed., University of Michigan Press, Ann Arbor, 1969.

7. J. Sally and W. Vasconcelos, Stable Rings, J. Pure \& Applied Algebra, 4 (1974), 319-336.

8. R. Wiegand, Dimension Functions on the Prime Spectrum, (Preprint).

Received February 20, 1975 and in revised form September 25, 1975. 



\section{Pacific Journal of Mathematics}

\section{Vol. 62, No. $1 \quad$ January, 1976}

Mieczyslaw Altman, Contractor directions, directional contractors and

directional contractions for solving equations . .................. 1

Michael Peter Anderson, Subgroups of finite index in profinite groups .........

Zvi Arad, Abelian and nilpotent subgroups of maximal order of groups of odd order

John David Baildon and Ruth Silverman, On starshaped sets and Helly-type theorems ..........................................

John W. Baker and R. C. Lacher, Some mappings which do not admit an

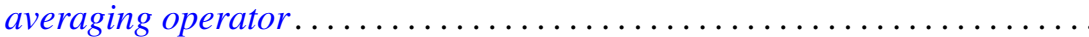

Joseph Barback, Composite numbers and prime regressive isols . . . . . . . . . .

David M. Boyd, Composition operators on $H^{p}(A) \ldots \ldots \ldots \ldots \ldots \ldots \ldots$

Maurice Chacron, Co-radical extension of PI rings . . . . . . . . . . . . .

Fred D. Crary, Some new engulfing theorems . . . . . . . . . . . . . . .

Victor Dannon and Dany Leviatan, A representation theorem for convolution transform with determining function in $L^{p} \ldots \ldots \ldots \ldots \ldots \ldots \ldots \ldots \ldots \ldots \ldots \ldots \ldots \ldots$

Mahlon M. Day, Lumpy subsets in left-amenable locally compact

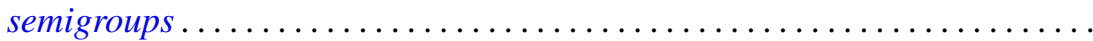

Michael A. Gauger, Some remarks on the center of the universal enveloping algebra of a classical simple Lie algebra . .

David K. Haley, Equational compactness and compact topologies in rings

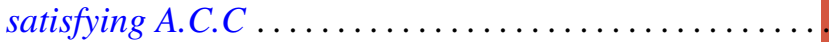

Raymond Heitmann, Generating ideals in Prüfer domains .

Gerald Norman Hile, Entire solutions of linear elliptic equations with

Laplacian principal part. .

Richard Oscar Hill, Moore-Postnikov towers for fibrations in which $\pi_{1}$ (fiber) is non-abelian

John Rast Hubbard, Approximation of compact homogeneous maps . .

Russell L. Merris, Relations among generalized matrix functions . .

V. S. Ramamurthi and Edgar Andrews Rutter, On cotorsion radicals ...

Ralph Tyrrell Rockafellar and Roger Jean-Baptiste Robert Wets, Stochastic

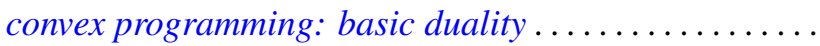

Alban J. Roques, Local evolution systems in general Banach spaces ..

I. Bert Russak, An indirect sufficiency proof for problems with bounded state variables.

Richard Alexander Sanerib, Jr., Ultrafilters and the basis property. .

H. A. Seid, The decomposition of multiplication operators on $L_{p}$-spaces . .

Franklin D. Tall, The density topology .................. 